\title{
A Plasmonic DNAzyme Strategy for Point-of- Care Genetic Detection of Infectious Pathogens
}

\author{
Kyryl Zagorovsky and Warren C. W. Chan \\ Version Post-Print/Accepted Manuscript \\ Citation K. Zagorovsky, W. C. W. Chan, “A Plasmonic DNAzyme Strategy for \\ (published version) Point-of-Care Genetic Detection of Infectious Pathogens," \\ Angewandte Chemie International Edition, 2013, 52, 3168 JIF =13.7. \\ Publisher's Statement This is the peer reviewed version of the following article: K. \\ Zagorovsky, W. C. W. Chan, "A Plasmonic DNAzyme Strategy for \\ Point-of-Care Genetic Detection of Infectious Pathogens," \\ Angewandte Chemie International Edition, 2013, 52, 3168 JIF =13.7., \\ which has been published in final form at \\ http://dx.doi.org/10.1002/anie.201208715. This article may be used for \\ non-commercial purposes in accordance with Wiley Terms and \\ Conditions for Self-Archiving.
}

\section{How to cite TSpace items}

Always cite the published version, so the author(s) will receive recognition through services that track citation counts, e.g. Scopus. If you need to cite the page number of the TSpace version (original manuscript or accepted manuscript) because you cannot access the published version, then cite the TSpace version in addition to the published version using the permanent URI (handle) found on the record page. 


\title{
A Plasmonic DNAzyme Strategy for Point-of-Care Genetic Detection of Infectious Pathogens**
}

\author{
Kyryl Zagorovsky, and Warren C. W. Chan*
}

The development of simple, cost-effective, sensitive, and specific point-of-care (POC) diagnostics represents a major challenge in the 21 st century. ${ }^{[1]}$ The spread of infectious diseases has caused significant losses in global economy and life. A method to control the spread of diseases is to detect the pathogens early at POC, and to administer proper treatment or quarantine. ${ }^{[2]}$ Detection of DNA as biomarkers is currently a widely used technique for pathogenic detection. ${ }^{[3]}$ Quantitative PCR (qPCR) is the method of choice, since it includes an enzymatic signal amplification step to achieve highly sensitive and specific detection of genetic targets. ${ }^{[4]}$ However, qPCR requires expensive equipment and highly trained personnel, limiting the technique use to medical laboratories. There is a strong need to develop cheaper and simpler DNA detection methods. ${ }^{[5]}$ Here, we combined colorimetric coupling of surface plasmons of gold nanoparticles with DNAzyme signal amplification technology to engineer a fast and simple detection platform for genetic targets with a simple colorimetric readout. The novelty of this study is the integration of these two emerging technologies for point-of-care analysis of infectious disease targets.

DNAzyme is a synthetic DNA enzyme that can catalyze the cleavage of another nucleic acid molecule. ${ }^{[6-8]}$ Since the catalysis is carried out with multiple turnover, DNAzyme introduces an enzymatic amplification step into the experimental setup. ${ }^{[9]}$ This amplification is performed without the need for protein components, which are costly and have low thermal and storage stability. While initially designed to detect $\mathrm{Pb}^{2+}$ and other divalent cations, ${ }^{[10-19]} \mathrm{a}$ number of modifications have been reported that allow DNAzyme to detect biomolecular targets. ${ }^{[9,20-27]}$ Two main approaches were used to implement detection of genetic targets. The first method utilizes competitive activation of peroxidase-mimicking DNAzyme, which can catalyze production of colorimetric or chemiluminescent product. ${ }^{[28]}$ In the second, more sensitive approach, a similar strategy was followed by a number of groups to implement detection of genetic targets by splitting the DNAzyme into inactive components, which could be reactivated by the target binding. ${ }^{[9,23-}$ ${ }^{25,29]}$ However, these studies used fluorescence as the readout, which

[*] K. Zagorovsky, Dr. W. C. Chan

Institute of Biomaterials and Biomedical Engineering,

Terrence Donnelly Centre for Cellular and Biomolecular Research, university of Toronto

160 College Street, Room 450, Toronto, ON, Canada M5S 3E1

E-mail: warren.chan@utoronto.ca

[**] The authors acknowledge the Canadian Institute of Health Research (MOP93532) and Natural Science and Engineering Research Council (CRDPG411601) for funding through the CHRP program (CPG-112321), Canadian Foundation for Innovation, and Ministry of Research and Innovation. The authors thank Mr. Leo Chou for pertinent discussions, and Dr. Anu Rebbapragada and Mr. Stephen Perusini for designing the targets for $N$. gonorrhoeae detection.

Supporting information for this article is available on the WWW under http://www.angewandte.org or from the author is not an optimal detection modality for POC applications since it requires access to fairly complex fluorometer apparatus. An alternative method using the colorimetric readout of gold nanoparticles (GNPs) has been reported for detection of metal ions, adenosine and cocaine..$^{[10-14,16-18,20,21]}$ The wavelength at which GNPs absorb light is dependent on whether they are in a monodispersed or aggregated state. ${ }^{[30]}$ Since the GNP solutions in monodispersed or aggregated states are easily distinguishable by their respective red or purple colors, this approach provides clear colorimetric results that can be visualized by the naked eye. ${ }^{[31]}$ In this study we combined DNAzyme technology with GNPs to engineer a simple point-of-care diagnostic platform that can be used in remote settings. ${ }^{[32,33]}$

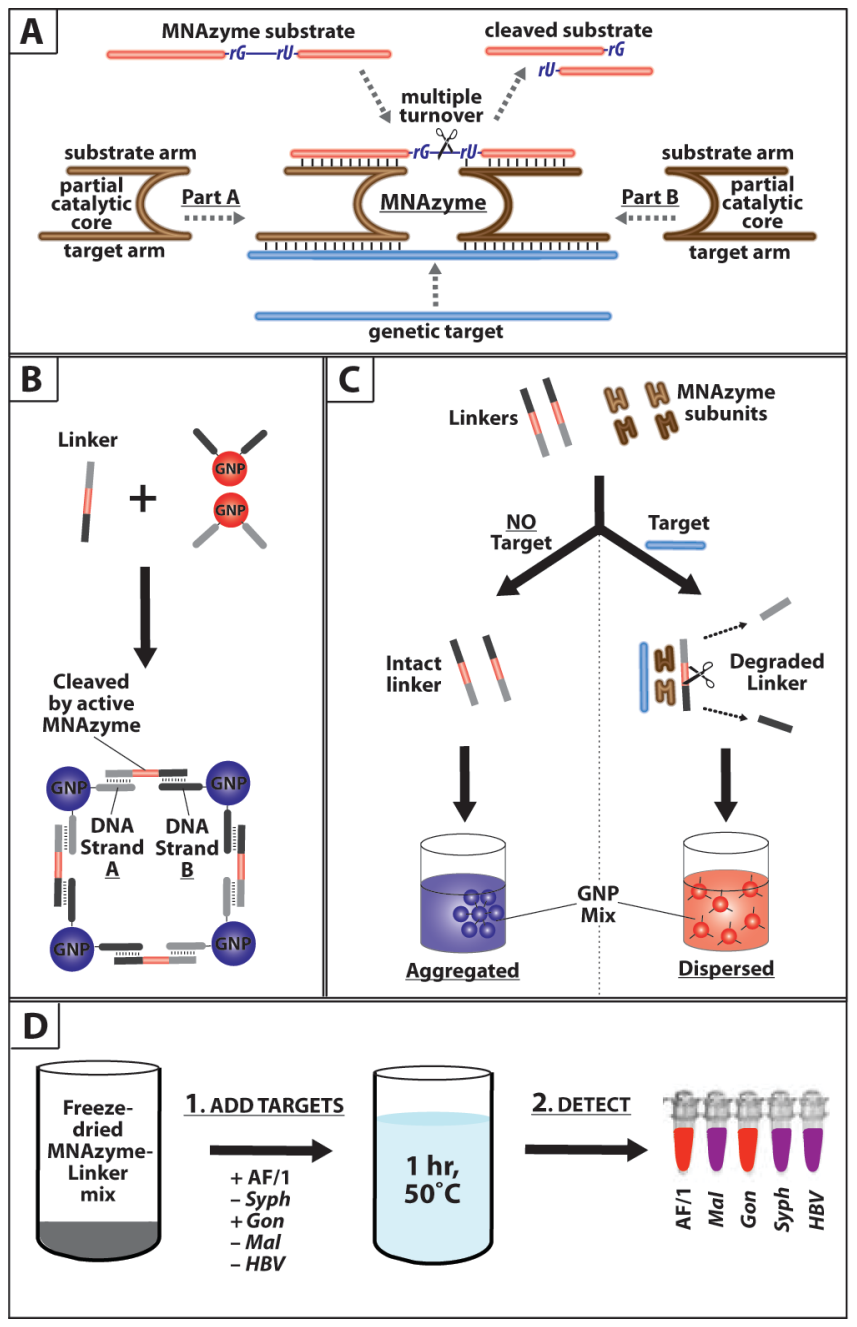

Figure 1. A - Mechanism of MNAzyme catalysis. Adapted with permission from [9]. Copyright 2009 American Chemical Society. $\underline{B}-$ GNP aggregate formation. Linker hybridizes to DNA strands $A$ and $B$, cross-linking the two sets of GNPs and turning solution purple. $\underline{\mathbf{C}}-$ MNAzyme assay outline. Target activated MNAzyme degrades Linker DNA, preventing GNP aggregate formation. In the absence of the 
target Linkers remain intact and cross-link GNPs (as in B); the solution turns purple. $\underline{\mathbf{D}}$ - Schematic depicting how the assay will be conducted at point-of-care to analyze multiple targets in parallel.

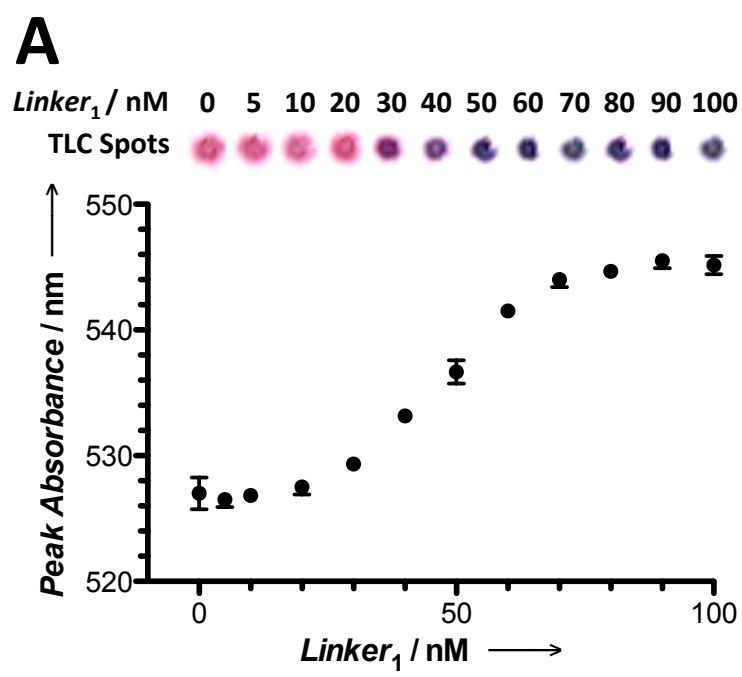

B

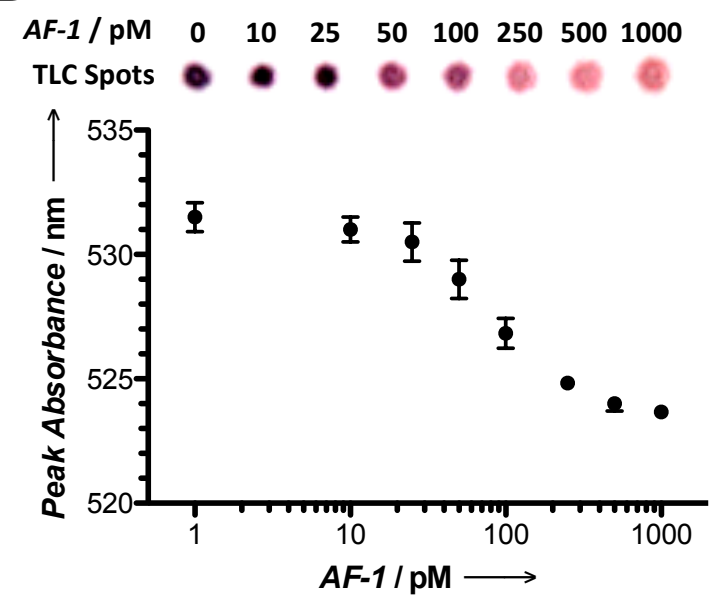

C

$\underline{\text { Targets Detected }}$

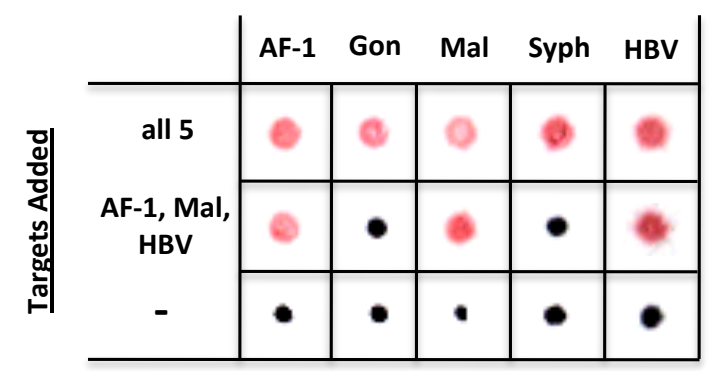

Figure 2. A - Optimization of Linker ${ }_{1}$ concentration. B - Sensitivity of detecting AF-1 genetic target. A and $\mathbf{B}$ - Top: colorimetric readout on TLC plate. Bottom: shift in the peak absorbance. Data based on 3 replicates; error bars are standard errors. C - Parallel detection of 5 targets: AF-1, genetic sequences from N. gonorrhoeae (Gon) and $T$. pallidum (Syph) bacteria, malarial parasite $P$. falciparum (Mal), and $H B V$ virus. Detection of all 5 targets (top row), 3 out of 5 targets (center row), or no-target control (bottom row). Spot color indicates target presence (red) or absence (purple-black). Images were adjusted for contrast and brightness.
Figure 1 demonstrates the molecular details of experimental setup. Two sets of $13 \mathrm{~nm}$ GNPs, GNP-A and GNP-B, are functionalized with 5'-thiol-modified Agg/A (Strand A) or 3'-thiolmodified Agg/B (Strand B) DNA, respectively. ${ }^{[34]}$ Linker strand is designed to have its 5'-end complementary to 3' of Agg/A, and its 3'-end to complement 5' of Agg/B. Linker can cross-link GNP-A and GNP-B through hybridization, forming GNP aggregate network (Figure 1-B). Close proximity of linked GNPs causes coupling of their individual localized plasmon fields, leading to the shift in their peak absorbance to a longer wavelength, and solution color changes from red to purple. In addition, the Linker strand includes the substrate sequence that can be cleaved by the multicomponent nucleic acid enzyme, MNAzyme, which is one of the reported DNA-responsive DNAzymes. Initially in the inactive state, target's binding to the sensor arms bridges MNAzyme parts A and B, forming the active structure, which can then hybridize to the substrate sequence and catalyze its cleavage (Figure 1-A). ${ }^{[9]}$ The substrate sequence comprises DNA bases with two RNA nucleotides, which are required for cleavage, imbedded in the middle. ${ }^{[6]}$ MNAzyme can be made responsive to any genetic target simply by changing the DNA bases of the sensor arms. The sequence of the substrate arms ensures MNAzyme's specificity to particular substrate, so that multiple MNAzyme-substrate pairs can be used in the same assay without cross-reactivity. MNAzyme uses $\mathrm{Mg}^{2+}$ as the divalent cation, and has optimal activity at $50^{\circ} \mathrm{C}$. Among the three reported DNA-responsive bipartite DNAzymes, we chose to work with the MNAzyme, since it has the highest catalytic rate. ${ }^{[9,24,25,29]}$

The genetic sensing assay is implemented as a simple two-step system (Figure 1-C). In the first "amplification" step, a buffered mix of MNAzymes and Linkers is added to the sample solution and incubated at $50^{\circ} \mathrm{C}$ for 1 hour. If genetic targets are present, they bind and activate the MNAzymes. Each active MNAzyme in turn catalyzes cleavage of multiple Linkers, which effectively translates into signal amplification. In the second "detection" step, a mix of GNP-A and GNP-B is added to the sample solution. In the absence of genetic target intact Linkers interact with the GNPs to form aggregate network, turning solution purple. In contrast, presence of genetic target results in Linker degradation. GNP-A and GNP-B do not get cross-linked, and solution remains red. For readout we use the change in solution color by spotting $3 \mu \mathrm{L}$ of solution onto thin layer chromatography (TLC) plates. ${ }^{[31]}$ TLC plates intensify the the color differences allowing for a more sensitive visual detection, and can be stored for later viewing. A measurement of the UV-vis spectroscopic shift of peak absorbance is used for confirmation. ${ }^{[35]}$

One critical parameter to optimize for the assay is the amount of Linker strand to use. For a given GNP concentration there is a minimal Linker concentration able to crosslink the GNPs sufficiently to cause visual aggregation. With such a Linker/GNP ratio, even if a small proportion of Linker strands get cleaved by the MNAzymes, this should have an effect on GNP crosslinking state and color of the solution. Therefore, minimizing the Linker concentration effectively maximizes assay sensitivity. Optimization results are presented in Figure 2-A. Assay setup matched the experimental condition of the MNAzyme ${ }_{1}$ assay, but lacked the genetic target to prevent Linker $_{1}$ degradation. As the amount of Linker $_{1}$ is increased from 0 to $100 \mathrm{nM}$, there is a sigmoidal shift in the peak absorbance, $\lambda_{\text {Peak }}$, from $527 \mathrm{~nm}$ to $546 \mathrm{~nm}$, indicating increase in GNP aggregate size. The TLC spot color follows a similar, but steeper sigmoidal trend. The spots appear red up to 20 $\mathrm{nM}$, than gradually shift to dark purple-black at $50 \mathrm{nM}$, and remain unchanged for higher concentrations. Based on both color change and peak shift we chose $40 \mathrm{nM}$ as the optimal Linker ${ }_{1}$ concentration.

Next, we carried out the MNAzyme assay outlined in Figure 1 to detect AF-1 as the DNA target. As determined previously, the 
Linker at $40 \mathrm{nM}$ was used, and we assayed $\mathrm{AF}-1$ from $0 \mathrm{pM}$ to $1 \mathrm{nM}$ (Figure 2-B). Similar to Figure 2-A, spectroscopic data demonstrates a sigmoidal, but decreasing trend in $\lambda_{\text {Peak }}$. Peak absorbance shifts from $533 \mathrm{~nm}$ for the sample without AF-1 to $523 \mathrm{~nm}$ for $1 \mathrm{nM}$ of target. The color of TLC spots follows similar trend, remaining purple from $0 \mathrm{pM}$ to $25 \mathrm{pM} \mathrm{AF-1}$, then gradually shifting to reach red at $500 \mathrm{pM}$ and $1000 \mathrm{pM}$. Both spectroscopic and spot color data establish the limit of detection of this assay at $50 \mathrm{pM}$. This sensitivity is slightly lower than $5 \mathrm{pM}$ reported for the fluorescence readout. ${ }^{[9]}$

Interestingly, if the Linker is chosen as the genetic detection target instead of AF-1, the experiment of Figure 2-A matches the original MNAzyme-free direct DNA detection assay reported by Mirkin group (we confirmed that the $50^{\circ} \mathrm{C}, 1$ hour incubation step does not affect assay results, Figure S1). ${ }^{[1]}$ Based on Figure 2-A, our implementation of this assay has detection sensitivity of $\sim 30 \mathrm{nM}$. Therefore, inclusion of MNAzyme enzymatic signal amplification step increases detection sensitivity by a factor of 600 .

We further investigated whether MNAzyme colorimetric assay can detect multiple targets in parallel (Figure 1-D). We chose 4 additional targets corresponding to genetic sequences for gonorrhea $(\mathrm{Gon})^{[36]}$ and syphilis $(\mathrm{Syph})^{[37]}$ bacteria, malaria parasite $(\mathrm{Mal}),{ }^{[38]}$ and hepatitis $\mathrm{B}$ virus $(H B V) .{ }^{[39]}$ These infectious diseases are prevalent in both the developed and developing world, and can cause severe health problems and death in patients that are not properly diagnosed and treated. To detect these targets, 4 new MNAzyme-Linker-GNP sets were developed, each specific for one of the targets. For example, MNAzyme Mal $_{\text {had substrate arms }}$ specific for Linker ${ }_{M a l}$, and sensor arms complementary to Mal target, but not other Linker sequences or targets. Linker $_{M a l}$, in turn, could aggregate GNP-A $\mathrm{A}_{\text {Mal }}$ and GNP-B $\mathrm{B}_{\text {Mal }}$, but not other GNP pairs. Another modification introduced was lyophilization of assay components. In this form reagents remain functionally stable during storage and transport, making them well suited for POC testing. ${ }^{[40]}$

In the first step of the assay, solution containing genetic targets is added to a reaction tube containing a mixture of 5 sets of MNAzyme-Linker pairs, and incubated at $50^{\circ} \mathrm{C}$ for 1 hour. Assuming AF-1 and Gon targets are present, they activate MNAzyme $_{\mathrm{AF}-1}$ and MNAzyme ${ }_{\text {Gon }}$, which in turn degrade Linker $_{\mathrm{AF}-1}$ and Linker ${ }_{G o n}$. The other 3 Linkers remain intact. In the second step, $10 \mu \mathrm{L}$ of the MNAzyme-Linker-target solution is transferred to 5 detection tubes, each containing target-specific GNP pair. Any Linkers that remain un-cleaved aggregate the corresponding GNP pairs, turning solution purple. In contrast, Linkers cleaved by targetactivated MNAzymes are not able to cross-link associated GNP pairs, and solution remains red. In the example above, only mixtures in AF-1 and Gon-specific detection tubes would remain red. Following this protocol we successfully detected all 5 targets simultaneously (Figure 2-C). In addition, the assay with lyophilized components was used to correctly identify $\mathrm{AF}-1, \mathrm{Mal}$ and $\mathrm{HBV}$ sequences in sample mixture contained 3 out of 5 targets.

In summary, MNAzyme-GNP assay provides a simple and fast colorimetric scheme for detection of genetic targets of bacterial, viral and fungal origins with $50 \mathrm{pM}$ sensitivity without the need for purification and separation steps. Results can be archived when spotted on TLC plates. To our knowledge, this is the first report of combining the multi-component DNA-responsive DNAzyme with gold nanoparticle colorimetric platform. Assay can detect multiple genetic sequences in parallel and is easily translatable to new nucleic acid targets. Color-based readout that does not require any complex equipment, and uses stable and cost-effective reagents make this experimental approach particularly suitable for POC testing. Furthermore, it is difficult to identify specific requirements for analytical sensitivity of a pathogen as this may vary based on the treatment strategy and whether one analyzing a single or coinfection. For more sensitive analysis, this POC system may require further tuning. Improved sensitivity could potentially be achieved by modifying the nanoparticle size and chemistry, varying the length of MNAzyme/Linker or target sequences ${ }^{[41]}$ or by combining the fast catalytic rate of MNAzyme and colorimetric plasmonic readout with the sensitive protein-free autocatalytic DNAzyme approach demonstrated by the Willner group. ${ }^{[23,42]}$ Furthermore, in a complete point-of-care system, we will likely need to include extra components that can extract the genetic targets of interest. Such technologies have been developed for isolating targets from blood and stool samples. ${ }^{[43-45]}$ Finally, synthetic genetic targets are commonly used as first step in the assessment of new POC devices. In the next step, further evaluation using clinical samples is required to assess clinical specificity and sensitivity prior to real-world use.

\section{Experimental Section}

Experimental details and supplementary figures can be found in the supplement.

Received: ((will be filled in by the editorial staff))

Published online on ((will be filled in by the editorial staff))

Keywords: nanoparticles · enzyme catalysis - biosensors · surface plasmon resonance $\cdot$ DNAzyme

[1] S. Binder, A. M. Levitt, J. J. Sacks, J. M. Hughes, Science 1999, 284, 1311-1313.

[2] T. S. Hauck, S. Giri, Y. Gao, W. C. W. Chan, Adv. Drug Delivery Rev. 2010, 62, 438-448.

[3] J. Weile, C. Knabbe, Anal. Bioanal. Chem. 2009, 394, 731-742.

[4] M. J. Espy, J. R. Uhl, L. M. Sloan, S. P. Buckwalter, M. F. Jones, E. A. Vetter, J. D. C. Yao, N. L. Wengenack, J. E. Rosenblatt, F. R. Cockerill III, T. F. Smith, Clin. Microbiol. Rev. 2006, 19, 165-256.

[5] S. Giri, E. A. Sykes, T. L. Jennings, W. C. W. Chan, ACS Nano 2011, $5,1580-1587$.

[6] K. Schlosser, Y. Li, ChemBioChem 2010, 11, 866-879.

[7] S. W. Santoro, G. F. Joyce, Proc. Natl. Acad. Sci. U. S. A. 1997, 94 , 4262-4266.

[8] R. R. Breaker, G. F. Joyce, Chemistry \& biology 1994, 1, 223-229.

[9] E. Mokany, S. M. Bone, P. E. Young, T. B. Doan, A. V. Todd, J. Am. Chem. Soc. 2010, 132, 1051-1059.

[10] W. Zhao, J. C. F. Lam, W. Chiuman, M. A. Brook, Y. Li, Small 2008, 4, 810-816.

[11] J. Liu, Y. Lu, J. Am. Chem. Soc. 2004, 126, 12298-12305.

[12] J. Liu, Y. Lu, J. Am. Chem. Soc. 2003, 125, 6642-6643.

[13] J. Liu, Y. Lu, Org. Biomol. Chem. 2006, 4, 3435-3441.

[14] D. Mazumdar, J. Liu, G. Lu, J. Zhou, Y. Lu, Chem. Commun. 2010, 46, 1416.

[15] X. Miao, L. Ling, X. Shuai, Chem. Commun. 2011, 47, 4192.

[16] H. Wei, B. Li, J. Li, S. Dong, E. Wang, Nanotechnology 2008, 19, 095501.

[17] J. H. Lee, Z. Wang, J. Liu, Y. Lu, J. Am. Chem. Soc. 2008, 130, $14217-14226$.

[18] Z. Wang, J. H. Lee, Y. Lu, Adv. Mater. 2008, 20, 3263-3267.

[19] J. Li, Y. Lu, J. Am. Chem. Soc. 2000, 122, 10466-10467.

[20] J. Liu, Y. Lu, Anal. Chem. 2004, 76, 1627-1632.

[21] J. Liu, Y. Lu, J Fluoresc 2004, 14, 343-354.

[22] D. Wang, B. Lai, D. Sen, J. Mol. Biol. 2002, 318, 33-43.

[23] F. Wang, J. Elbaz, R. Orbach, N. Magen, I. Willner, J. Am. Chem. Soc. 2011, 133, 17149-17151.

[24] F. Wang, J. Elbaz, C. Teller, I. Willner, Angew. Chem. Int. Ed. 2010, 50, 295-299.

[25] Y. V. Gerasimova, E. Cornett, D. M. Kolpashchikov, ChemBioChem 
2010, 11, 811-817.

[26] S. Sando, A. Narita, T. Sasaki, Y. Aoyama, Org. Biomol. Chem. 2005, 3, 1002-1007.

[27] S. Sando, T. Sasaki, K. Kanatani, Y. Aoyama, J. Am. Chem. Soc. 2003, 125, 15720-15721.

[28] J. Kosman, B. Juskowiak, Anal. Chim. Acta 2011, 707, 7-17.

[29] D. M. Kolpashchikov, ChemBioChem 2007, 8, 2039-2042.

[30] M. Quinten, U. Kreibig, D. Schönauer, L. Genzel, Surf. Sci. 1985, $156,741-750$.

[31] R. Elghanian, J. J. Storhoff, R. C. Mucic, R. L. Letsinger, C. A. Mirkin, Science 1997, 277, 1078-1081.

[32] C. D. Medley, J. E. Smith, Z. Tang, Y. Wu, S. Bamrungsap, W. Tan, Anal. Chem. 2008, 80, 1067-1072.

[33] K. Sato, K. Hosokawa, M. Maeda, Nucleic Acids Res. 2005, 33, 1, e4.

[34] S. J. Hurst, A. K. R. Lytton-Jean, C. A. Mirkin, Anal. Chem. 2006, 78, 8313-8318.

[35] J. J. Storhoff, R. Elghanian, R. C. Mucic, C. A. Mirkin, R. L. Letsinger, J. Am. Chem. Soc. 1998, 120, 1959-1964.

[36] R. Rossau, L. Heyndrickx, H. Van Heuverswyn, Nucleic Acids Res. $\mathbf{1 9 8 8}, 16,6227$.

[37] J. Burstain, E. Grimprel, S. Lukehart, M. Norgard, J. Radolf, J. Clin.
Microbiol. 1991, 29, 62-69.

[38] A. R. Bharti, K. P. Patra, R. Chuquiyauri, M. Kosek, R. H. Gilman, A. Llanos-Cuentas, J. M. Vinetz, Am. J. Trop. Med. Hyg. 2007, 77, 444446.

[39] D. Paraskevis, A. Beloukas, C. Haida, A. Katsoulidou, Z. Moschidis, H. Hatzitheodorou, A. Varaklioti, V. Sypsa, A. Hatzakis, Virol. J. 2010, 7, 57.

[40] W. Abdelwahed, G. Degobert, S. Stainmesse, H. Fessi, Adv. Drug Delivery Rev. 2006, 58, 1688-1713.

[41] Y. Gao, W. L. Stanford, W. C. W. Chan, Small 2011, 7, 137-146.

[42] S. Shimron, F. Wang, R. Orbach, I. Willner, Anal. Chem. 2012, 84, 1042-1048.

[43] S. R. Jangam, D. H. Yamada, S. M. McFall, D. M. Kelso, J. Clin. Microbiol. 2009, 47, 2363-2368.

[44] A. V. Govindarajan, S. Ramachandran, G. D. Vigil, P. Yager, K. F. Boehringer, Lab Chip 2012, 12, 174-181.

[45] A. G. Freifeld, K. A. Simonsen, C. S. Booth, X. Zhao, S. E. Whitney, T. Karre, P. C. Iwen, H. J. Viljoen, The Journal of Molecular Diagnostics 2012, 14, 274-279. 
Entry for the Table of Contents (Please choose one layout)

Layout 2:

\section{Plasmonic Biosensor}

Kyryl Zagorovsky, Warren C. W. Chan* Page - Page

A Plasmonic DNAzyme Strategy for Point-of-Care Genetic Detection of Infectious Pathogens

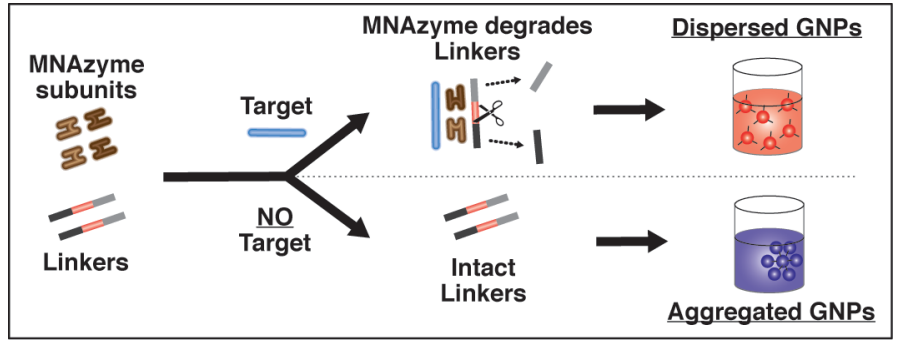

DNAzyme signal amplification is combined with surface plasmon properties of gold nanoparticles to achieve simple and sensitive colorimetric detection of genetic targets. The assay has $50 \mathrm{pM}$ sensitivity without the need for purification steps, can detect multiple targets in parallel, and is easily adaptable to new targets. This technology is capable of rapid detection of genetic targets for gonorrhea, syphilis, malaria, and hepatitis B infections. 